WellBeing International

WBI Studies Repository

10-1999

\title{
Predicting Flock Vigilance from Simple Passerine Interactions: Modelling with Cellular Automata
}

\author{
David B. Bahr \\ University of Colorado \\ Marc Bekoff \\ University of Colorado
}

Follow this and additional works at: https://www.wellbeingintlstudiesrepository.org/acwp_ena

Part of the Animal Studies Commons, Behavior and Ethology Commons, and the Comparative Psychology Commons

\section{Recommended Citation}

Bahr, D. B., \& Bekoff, M. (1999). Predicting flock vigilance from simple passerine interactions: modelling with cellular automata. Animal Behaviour, 58(4), 831-839.

This material is brought to you for free and open access by WellBeing International. It has been accepted for inclusion by an authorized administrator of the WBI Studies Repository. For more information, please contact wbisr-info@wellbeingintl.org.

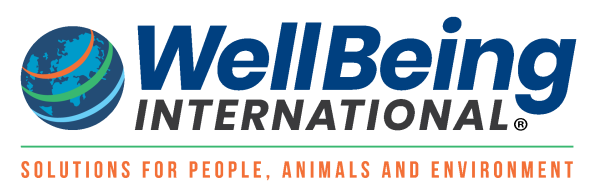




\title{
Predicting Flock Vigilance from Simple Passerine Interactions: Modelling with Cellular Automata
}

David B. Bahr and Marc Bekoff

University of Colorado

\begin{abstract}
Vigilance in flocks can be described and modelled as a plausible set of local interactions between neighbouring birds. Each bird in the modelled flock chooses to feed or to scan based solely on whether or not its neighbours are feeding or scanning. This simple model has the ability both to reproduce observations that have not been previously explained and to predict flock behaviours that might be confirmed with future field studies. Examples include simulations showing decreased vigilance with increased flock size (as observed in the field), greater time spent scanning when obstacles such as trees are present (as observed) and a coordinated feed/scan pattern (that is predicted to become increasingly coordinated when the birds look up from feeding more frequently). The numerical model also predicts that flock geometry influences vigilance. If two flocks are the same size, individuals in the one with the larger perimeter will spend more time scanning. This prediction could be tested with field studies and already has been observed empirically for two limiting cases: birds arranged in a line (high perimeters, high scan times) and birds in a circle (lower perimeters, lower scan times). As demonstrated by its multiple successes, cellular models of this type are a powerful new approach to understanding bird flock behaviours.
\end{abstract}

Birds, mammals and fish display a wide range of remarkably coordinated behaviour. Birds in a flock take turns eating and scanning for predators. Fish swim in schools that react in concert to external stimuli. Herd animals such as elk, deer, bison, sheep and cows move, change directions and even stampede in unison. Fireflies blink as if to a prearranged beat. Explanations for social behaviour, or why some animals live and act in groups, range from selfish herd hypotheses to risk dilution (Hamilton 1971; Elgar 1989; Lima 1990; Sadedin \& Elgar 1998) and typically centre on predator-prey relationships (better hunting or better predator deterrence in a group). Similarly, the advantages of having coordinated behaviours within a group can frequently be explained in terms of enhanced foraging, predator detection, or other necessities of survival. However, these explanations fail to describe the process that creates the coordinated behaviour. How can every member in a school of fish change directions simultaneously without some obvious cue? Or how can a flock of birds organize into distinct patterns where the birds trade turns scanning and eating? Frequently trees or other obstructions can block the view of a flock or herd, so how does each individual assess flock size and coordinate its scanning and feeding behavior accordingly with other flock members, as they are observed to do? 
For groups of people, common wisdom holds that coordinated behaviour requires a leader or some other cue that all individuals can follow. Intuitively, we believe, for example, that a marching band without a 'drum major' would hardly keep in step. On the other hand, flocks of birds and schools of fish can change direction in unison with no apparent leader. The birds (and fish and other animals) appear to make decisions and act as individuals but none the less behave in concert with the larger flock. Deriving the origins of such group behaviour would not only explain certain animal interactions, but also has the potential to explain apparently spontaneous interactions by large groups of humans that have no obvious leader (e.g. some riots and religious behaviours: Bahr \& Passerini 1998a, b).

If a group of people is lined up and told to march forward 'in step' with no further directions (i.e. nobody calling out 'left right left right'), each individual might try to glance up and down the line to find out which foot their neighbours were putting forward with each step. Based on what they see, each individual can decide whether or not they are in step with the rest of the group and can alter their march accordingly. In a short time, the group will synchronize and march in step, as easily verified with a few people. As with a flock of birds, each person appears to make decisions as an individual but is coordinated with the whole group. Analogous human behaviours are well documented, and include various fads, gawking, tipping strategies and store brand loyalty (e.g. Callen \& Shapero 1974; Latané 1981).

We hypothesize that a similar individual decision-making process underlies the coordinated feeding behaviour of birds in a flock. We postulate simply that birds in a flock decide whether to feed or to scan for predators based on the number of birds visible to them that are engaged in each behaviour. If more neighbours are feeding, then a bird is more likely to scan for predators. If more neighbours are scanning, then a bird is more likely to eat and take advantage of the group's vigilance. A numerical model based on this premise reproduces a wide variety of observed passerine behaviours. In particular, when each bird in a flock interacts with other birds according to this rule, the model predicts that vigilance decreases with flock size, that vigilance changes with flock geometry, and that birds coordinate and trade turns feeding and scanning (particularly when the birds are arranged in a square or circle). All of these behaviours have been noted with real flocks (e.g. Bekoff 1995).

\section{BACKGROUND}

Although a number of species display coordinated group behaviours, many, such as fish, are difficult to observe. Some researchers have successfully followed arboreal monkeys, canids, ostriches, Struthio camelus, ibex, Capra pyrenaica, and assorted African mammals (e.g. Alados 1985; Burger \& Gochfeld 1988, 1994; Elgar 1989; Treves 1998), but in part because local populations of birds are easily attracted to feeders, many of the more detailed studies of group (flock) behaviour have focused on evening grosbeaks, Coccothraustes vespertinus, Steller's jays, Cyanocitta stelleri, spotted turtle doves, Streptopelia chinensis, house sparrows, Passer domesticus, and other small passerines (e.g. Elgar et al. 1984; Elgar 1989; Lima 1990; Bekoff 1995; Roberts 1997; Krams 1998; Sadedin \& Elgar 1998).

Commonly observed traits of a flock include the percentage of time spent eating versus scanning (gathering information about other birds or being vigilant against predators; e.g. Bekoff 1995), the rate at which birds come and go (e.g. Roberts 1997), the influence of dominance and gender (e.g. Krams 1998), and the influence of flock size and geometry on each of these other properties (e.g. Bekoff 1995; Sadedin \& Elgar 1998). Vigilance is one of the most frequently examined characteristics of a flock, and the vigilance of each bird is known to decrease slowly and asymptotically with increasing group size. Observations also show that group geometry affects the time spent scanning, with birds in linear flocks (e.g. birds on a wire) being more vigilant than birds in flocks arranged in a 'circle' or 'square' (e.g. birds around the edge of a platform feeder). Obstacles like trees that impede the view from one bird to another are also known to increase the time spent scanning (e.g. Metcalfe 1984; Elgar 1989). The importance of 
'continuity' or unimpeded views has also been demonstrated with arboreal monkeys and other mammals (Quenette 1990; Treves 1998). The percentage of time spent scanning does not appear to change once flock sizes exceed roughly 10 birds (e.g. Elgar et al. 1984). Scanning and vigilance behaviour is relatively easy to identify because birds cannot move their eyes; and when birds look up from eating, they almost always move their heads and body positions to observe their neighbour's behaviour or to gather (scan) other information about their environment.

Less-common observations involve the spatial patterns of behaviour within a flock. Examples include the spatial distribution of feeding versus scanning birds, and the location of dominant birds within a flock (e.g. Krams 1998). Videos of western evening grosbeaks arranged in a circle demonstrate a spatial relationship in which the birds take turns feeding and scanning (data from Bekoff 1995). This scan/feed or up/down pattern is visible because the birds pivot down to eat and pivot up (and turn their heads) to scan. Point biserial correlations show that the up/down pattern is more pronounced in flocks arranged in a circle than in flocks arranged in a line (Bekoff 1995).

Each of these traits (vigilance as a function of flock size and geometry; the development of an up/down pattern) depends on remarkably coordinated behaviour by the group. In many cases a dominant bird is obvious, but in almost no case does this dominant (or any other bird) appear to guide the group. There is no evidence, for example, supporting a leader that directly controls a flock's vigilance. Without a leader, however, individuals must be making decisions that lead inherently to the coordinated behaviour. What decisions can they make? Various studies have demonstrated that birds are capable of remembering food locations and constructing mental maps of their surroundings (Speakman 1987; Bingman 1993). Birds can delineate objects by categories and concepts (Pepperberg 1990), and similarly, some data show that birds can count or subitize (estimate the number of surrounding objects without explicitly counting; Pepperberg 1994; Bekoff 1996) and remember about seven different objects (such as the feeding or scanning behaviour of nearby birds; Emmerton \& Delius 1993). Although not tremendously complicated, these individual decisions form the basis of the model presented in the following section. In fact, only the ability to count or subitize and to remember the behaviour of their neighbouring birds is necessary to show that many of the coordinated vigilance and other behaviours described above follow as a consequence.

\section{THE MODEL}

To simulate the feeding and scanning behaviour of birds in as simple a manner as possible, we assume that each member of a flock occupies a site on a square grid (Fig. 1). Each bird can either eat, or look up and scan its eight nearest neighbours on the grid. In accordance with known cognitive skills, we assume that the birds can count or subitize the number of neighbours that are eating versus scanning. In the basic implementation of the model, each individual bird chooses to scan for predators if four or more (a majority) of its neighbours are eating (i.e. somebody had better scan). Similarly, a bird chooses to eat if a majority of its neighbours are already scanning (i.e. take advantage of the neighbour's vigilance). When eating, a bird will choose periodically to look up and scan its neighbours; this is done with a frequency specified as a model parameter. These assumptions are simple, plausible, frequently observed, and well within the ability of passerines. Although the remainder of this section describes more details, these basic interactions summarize the foundation of the model.

In the model, time is discretized into uniform steps (representing a few seconds, or some other reasonable length). At each time step of the model, the birds that are currently scanning or have just looked up from feeding will examine their eight neighbours and decide whether to continue scanning or return to eating (Fig. 1). This decision is made by following the previously described process of looking to see what the majority of birds is already doing. The model is initialized with a flock of a given size 
arranged in a specific geometry (e.g. linear, or along the outline of a square feeder). By starting with an initially random arrangement of feeding and scanning birds, the model advances through the time increments and keeps track of the percentage of birds that are scanning versus feeding. By plotting the arrangement of the birds at each time step, a computer video of their feeding behaviour can be observed for patterns in the flock's vigilance.

Figure 1. An example of two successive time steps in the model. A grid represents the locations of birds that are eating (black) or scanning (white). At time step 1, five birds are scanning and four are feeding. At time step 2, the birds have changed their behaviour to do the opposite of whatever the majority of their neighbours were doing. The bird in the centre, for example, saw that the majority of its neighbours were scanning, so it decided to continue eating. At the bottom-right corner, the bird changed from eating (time step 1) to scanning (time step 2) because the majority of its neighbours were eating at time step 1 (more details of behaviour at the periphery are described in the text).
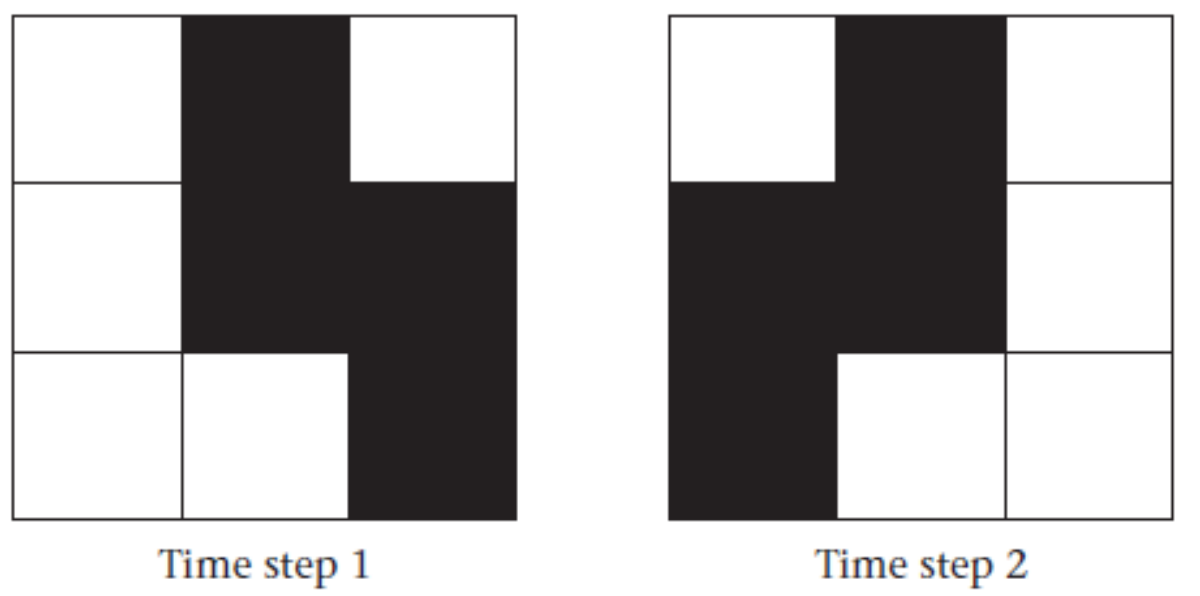

In practice, the computer code represents the flock as a square matrix of 1 s (scanning birds) and 0s (feeding birds). Based on the rules given above, each 1 or 0 is updated to another 1 or 0 at the next time step. When cast in this arrangement of $1 \mathrm{~s}$ and $0 \mathrm{~s}$, the model is easily identified as one of a very large class of so-called 'cellular automatons' (e.g. Wolfram 1986). These cellular models have a history of successful applications, such as explaining why zebra stripes can grow and creating simulations of particle physics (Young 1984). The 'majority rule' described above is a slightly modified version of a cellular automaton called the 'voter model' which is frequently used in statistical mechanics (e.g. Callen \& Shapero 1974).

In our simulations we made an important modification of the rules described above. Rather than deciding what to do based on the behaviour of the majority, the birds make a decision with a probability that is determined by the percentage of birds scanning. If no neighbours are scanning, then the bird will decide to scan with probability 1 (or 100\%). If all the neighbours are scanning, then the bird will decide to scan with probability 0 (or $0 \%$; in other words, the bird will definitely eat). If, however, half of the neighbours are scanning and the other half are eating, then the bird will decide to scan with probability 0.5 (or $50 \%$ ) and eat with probability 0.5 . In general, if $x$ per cent of a bird's neighbours are scanning, then there is an $x$ per cent chance that the bird will eat instead. In other words, the bird's decision is in part random. This is thought to represent the real cognitive process better, because we cannot say with exact certainty what a bird will do. Instead we can just say that a bird is more likely to scan or to eat given some observed behaviour of the surrounding birds. 
At the edges of the model grid (edges of the bird flock), each bird will have fewer than eight neighbours. Typically, a bird at the edge will have three or five neighbours, but in some geometries (e.g. a flock arranged in a line), the birds may have only one or two neighbours. As before, the birds choose to eat or scan based on the number of neighbouring birds that are already scanning; and just like birds in the interior of a flock, the probabilities are constructed as if the birds are looking for eight neighbours (as discussed above, this is roughly the total number of neighbours that a bird could count or observe, if that many were actually present). In the interior of a flock, each of the eight neighbours has an influence (or carries a weight) of one-eighth. For example, if five neighbours are scanning and three are eating, then the probability of scanning is three-eighths and the probability of eating is five-eighths. On the edges of a flock, the model rules do not change, and each neighbour still has an influence of only one-eighth. For example, if there is only one neighbour and it happens to be scanning, then the probability is one-eighth that the bird will eat and seven-eighths that the bird will scan. In general, if $n_{\text {scan }}$ is the number of neighbours that are scanning, then $n_{\text {scan }} / 8$ is the probability that the bird will eat instead. In other words, the rules are identical for both the boundary and the interior of the model grid.

Basically, the model assumes that birds on the boundary are trying to count (or subitize) the same number of neighbours (eight) as birds on the interior. One inevitable and immediate consequence of this assumption is that birds on the periphery will spend more time scanning. In essence, birds on the periphery cannot see (i.e. count or subitize) enough other birds that are scanning, so they will frequently choose to scan. As shown in the following sections, a number of predictions follow directly from the increased scanning at the edges of a flock. Also note that many observations support this behaviour of increased scanning at the edges (e.g. Elgar 1989; Bednekoff \& Ritter 1994; Hunter \& Skinner 1998).

Our current model does not incorporate the effects of bird gender, age, or dominance. Future studies could include these properties in the same way that similar cellular automata sociology models incorporate strong leaders, weak followers, and a whole array of other dominant versus subordinate interactions between human individuals (Bahr \& Passerini 1998a, b). (In these models, the dominant humans simply carry a weight greater than one-eighth, and the subordinate humans carry less weight.) Also, birds are not allowed to move around from place to place, or to come and go. Again, this could be changed. It is worth noting, however, that without these additional features, the bird model still reproduces many observed behaviours (as described in the following section). In our view, it is important to use the simplest model possible to explain the vigilance observations, and additional variables can be added later as necessary to explain other observations.

In summary, the model construction is straightforward. Birds in a flock are placed on a grid and allowed to interact with their nearest neighbours. At each time step the birds can decide to change from scanning to eating (and vice versa) with a probability that is proportional to the number of eight neighbours that are already scanning.

\section{RESULTS}

\section{Vigilance}

The model was run for a series of simulations with varying flock sizes and geometries. Each simulation was run for 20000 time steps with the birds looking up at every time step (except as noted, the fundamental results are largely unchanged when the birds look up less frequently). All interactions were between neighbouring birds, and nothing in the model was intentionally designed to encourage groupwide coordinated behaviour (e.g. no group leader). Nevertheless, the model does an excellent job of reproducing many empirical observations. 
Figure 2. The percentage of time spent scanning decreases with increasing flock size. Note that the asymptotic shape of the curves agrees with observations (e.g. Bekoff 1995; Sadedin \& Elgar 1998). Also note that most of the change happens for small flocks with fewer than roughly 10 birds, as noted in Elgar et al. (1984). (a) Birds arranged in linear flock sizes of 1-8. (b) Birds arranged in square-shaped flocks, as in Fig. 1, of 1, 4, 9, 16, 25, 36 and 100 individuals. (c) For unrealistically large square-shaped flocks, it is especially clear that the percentage of time spent scanning does not change significantly with increasingly large flock sizes. This plot contains flocks of sizes 1, 4, 9, 16, 25, 36, 100, 400, 900, 1600 and 10000.
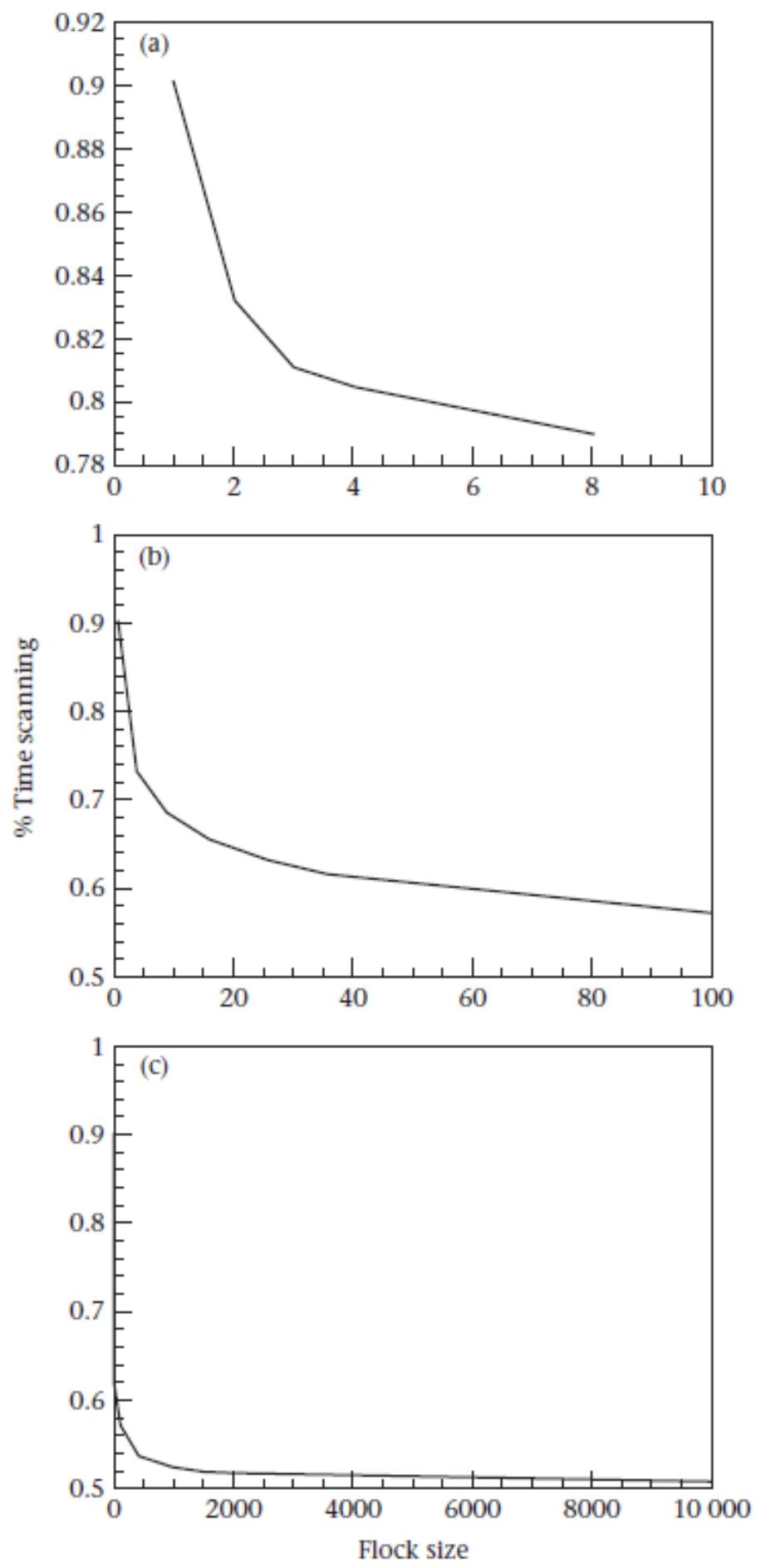
Consider, for example, the percentage of time spent scanning by a bird, measured as the number of time steps spent scanning divided by the total number of time steps in the model simulation. (For the numbers quoted here, we constructed a percentage for each bird and then averaged this over each bird in the flock.) The percentage of time spent scanning decreased asymptotically with increasing flock size (Fig. 2). For small flocks (four to six birds), the change was quite rapid as flock size increased. For larger flocks (roughly 10 or more birds), the percentage of time spent scanning continued to decrease with increasing flock size, but the rate of change slowed dramatically (in agreement with observations by Elgar et al. 1984). In the extreme of ridiculously large flocks (difficult to achieve in reality, but useful as a model result), the percentage of time spent scanning became essentially unchanged by an increase in flock size (Fig. 2).

Figure 3. The model predicts that larger perimeters and higher length-to-width ratios increase the percentage of time spent scanning. A square flock of birds, for example, will spend less time scanning than a flock arranged in a rectangle or line with the same number of birds. Each flock on these graphs contained 144 birds arranged into rectangles with different lengths and widths $(12 \times 12,6 \times 24,4 \times 36,3 \times 48,2 \times 72$, and $1 \times 144)$. (a) Scan time as a function of length/width. (b) Scan time as a function of perimeter (width+length).

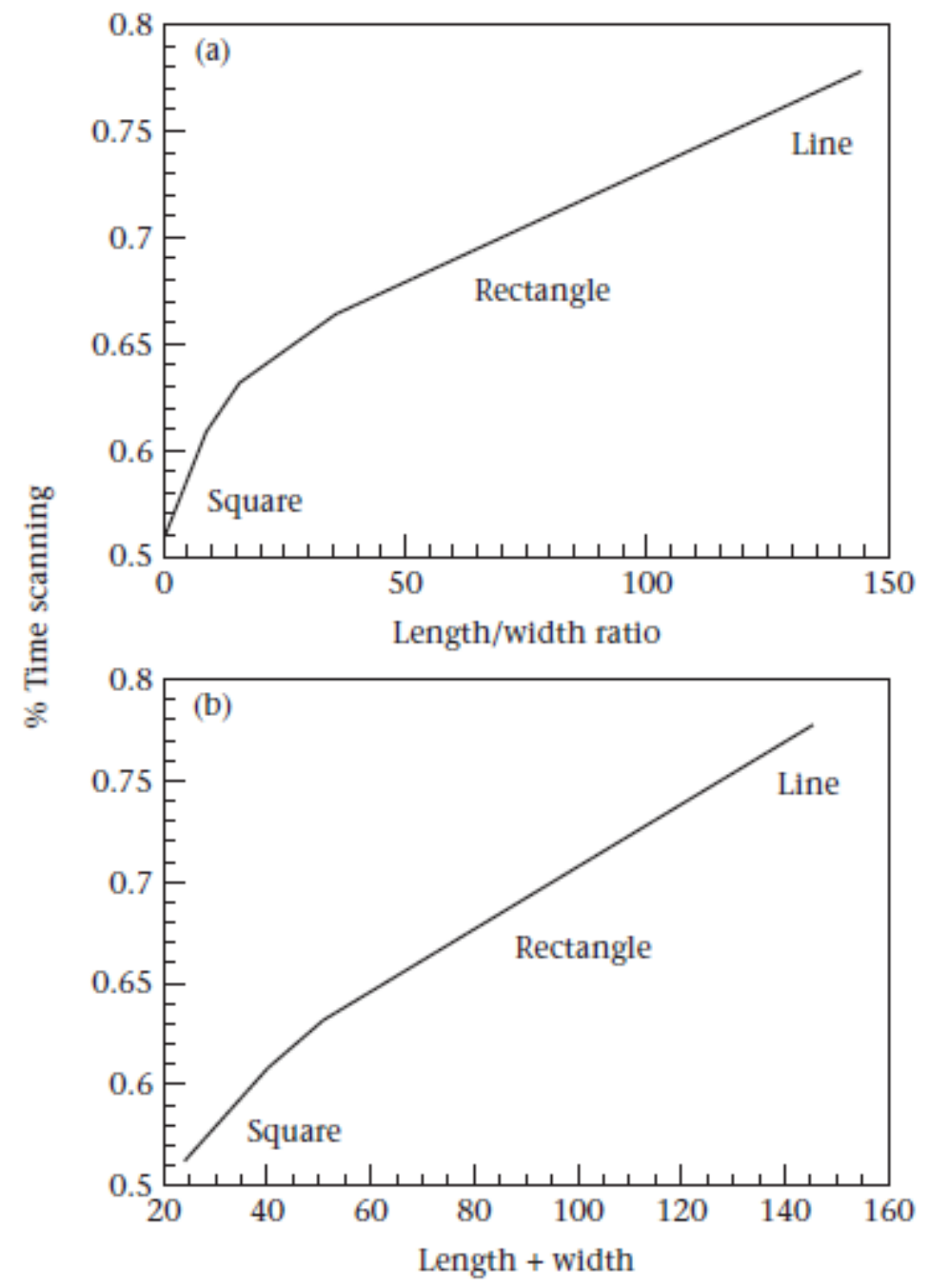

The model also predicted, in agreement with observation (e.g. Bekoff 1995), that group geometry affects vigilance. Birds in a line, which could only see two neighbours, spent more time scanning than the same 
number of birds arranged in a filled-in rectangle, which could see eight neighbours (Fig. 3). In fact, the model predicted as a simple extention of the assumptions that the time spent scanning is a function of the perimeter length or length-to-width ratio of the flock geometry. High ratios (longer perimeters) implied higher percentages of time spent scanning (Fig. 3). In other words, birds in geometries that are relatively stretched out (like a line or an elongated rectangle) spent on average more time scanning than the same number of birds that flocked compactly (like a filled square).

Figure 4. The mean number of neighbours for a bird increases with increasing flock size. Birds on the edge of a flock have fewer neighbours (which decreases the mean), but the influence of the perimeter is minimized as the flock size grows. Both the square- and linear-shaped flocks are plotted for group sizes of 4, 9, 16, 25, 36, 64 and 100 individuals.

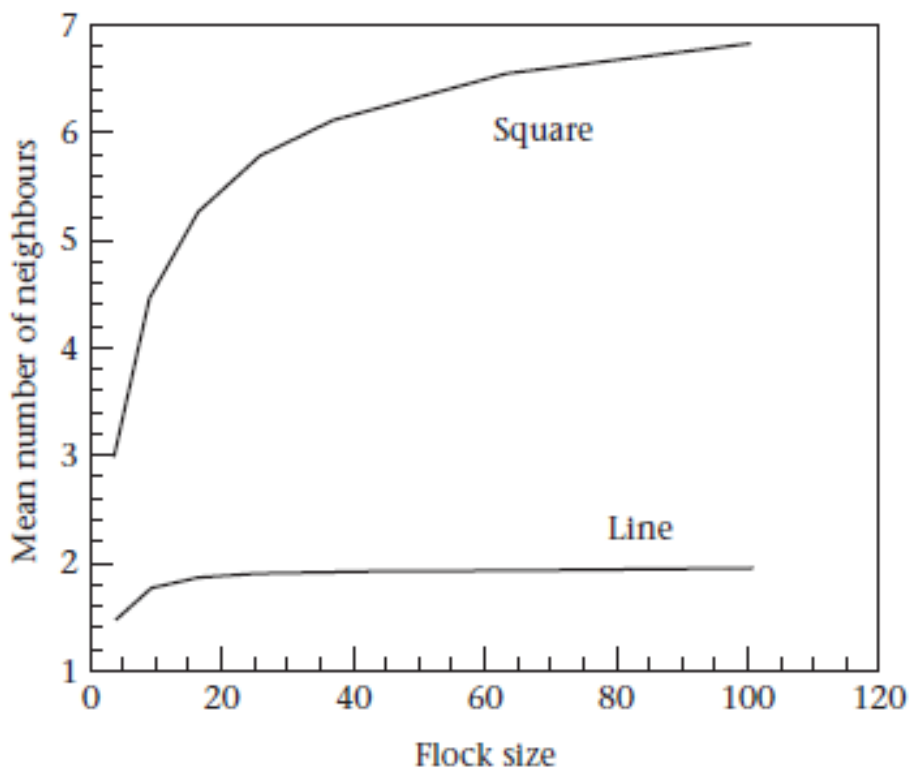

A simple explanation may partially explain the scan time's dependence on both the flock size and the length-to-width ratio. It seems plausible that the scan time will be strongly influenced by birds on the perimeter of the flock (Elgar 1989; Bednekoff \& Ritter 1994; Hunter \& Skinner 1998). These birds have fewer neighbours and therefore see fewer neighbours scanning (see description of the model above). As a result, birds on the perimeter are more likely to scan at any given time because no other bird is scanning for them (again, this follows from the assumption that the birds are capable of counting and are trying to count or subitize approximately eight neighbours). On the other hand, birds on the interior of the flock will scan less frequently because they have more neighbours, and these neighbours may already be scanning for them. In general, as illustrated by these differences on the edge versus the interior, the scan time decreases with increasing numbers of neighbours. Note that the mean number of neighbours for any bird will increase with increasing flock size because edge effects are minimized (Fig. 4), so on average the birds will spend less time scanning as the flock size grows. Alternatively, if we note that the percentage of scan time $(S)$ is proportional to the number of birds on the perimeter $\left(N_{\text {perimeter }}\right)$ and is inversely proportional to the number of birds in the flock $\left(N_{\text {total }}\right)$, then as a simple approximation

$$
S \propto N_{\text {perimeter }} / N_{\text {total }}
$$

This is an approximation to the mean number of neighbours, which for large square flocks is roughly $\left(5 N_{\text {perimeter }}+8 N_{\text {total }}\right) / N_{\text {total }}$, where 5 is the number of neighbours for a bird on an edge, and 8 is the 
number of neighbours for a bird on the interior. For large squares, the four additional vertices with three neighbours are negligible compared to $N_{\text {total }}$. For a square flock, the perimeter goes as the square root of the total number of birds. Therefore, $S \propto 1 \sqrt{ } N_{\text {total }}$. This is roughly the behaviour that was simulated with increasing flock sizes (Fig. 2). Also, when the total number of birds was fixed and only the geometry and perimeter changed, then $S \propto N_{\text {perimeter }}$, which is roughly demonstrated by the nearly straight line in Fig. $3 \mathrm{~b}$. Note that the prediction of increased vigilance with increased perimeter length could be tested explicitly in future field studies, although it is already consistent with observations showing that birds (and mammals) on the periphery spend more time scanning than birds in the interior of a flock (e.g. Elgar 1989; Bednekoff \& Ritter 1994; Hunter \& Skinner 1998).

\section{Coordinated Behaviour}

In both reality and model simulations, each bird in the flock is only capable of processing the behaviour of a limited number of neighbouring birds (Elgar et al. 1984). This does not preclude information being passed from bird to bird through the flock. Consider, for example, four birds in a flock that surround a tree. The bird on one side of the tree may see a predator and set off an alarm. The bird on the opposite side of the tree will not see the alarm because its view is blocked. However, the birds adjacent to the alarm will be warned and will flush. Once these birds have moved, the remaining bird on the far side of the tree will learn (second-hand through a chain of neighbours) that there is an alarm. So although an individual bird may not interact directly with the entire flock, information about unseen birds is slowly transmitted through a chain of neighbouring birds.

This chain of interactions is responsible for the coordinated scan/feed (or up/down) behaviour observed in the model simulations. Birds in the model will clump almost instantaneously (within a few time steps) into a pattern where the birds alternate eating and scanning (Fig. 5). The pattern tends to be patch-like, with birds in each patch sharing the same turns eating and scanning. The patches can change with time, growing and shrinking so that birds that once shared the same turns eating and scanning might now trade turns eating and scanning. This growing and shrinking of patches is part of the process of information transmission across the flock. For very large flocks, this coordinated scan/feed pattern is particularly obvious and is illustrated in Fig. 5. For smaller flocks, the scan/feed pattern is less obvious, but computergenerated videos of the simulations make the smaller flock's coordinated behaviour more visible.

Two model parameters significantly affect the extent of the coordinated scan/feed pattern. First, the more frequently the birds look up from eating to check on their neighbours, the more cooperative (synchronized) the flock's scan/feed behaviour becomes. In essence, the information transmission across the flock is impeded not only by trees and physical obstacles, but also by birds that never bother to look up and find out what their neighbouring birds are doing. In all of the simulations exploring vigilance (percentage of scan times) and most of the simulations involving the scan/feed pattern, we have specified that the birds look up frequently (at every time step).

Second, the coordinated scan/feed pattern is strongly influenced by the ease with which members of the flock are persuaded to change from scanning to eating or vice versa. This 'persuasiveness' is commonly included in cellular automata models as a parameter called 'social temperature' (Callen \& Shapero 1974; Bahr \& Passerini 1998a, b). (The name derives from the use of cellular automata models in physics where the same parameter is the physical temperature; Ma 1985; Huang 1987.) When temperatures are high, the birds are easily convinced to change (from scanning to eating or vice versa), and when temperatures are low, the birds are reluctant to change their current behaviour (sluggish or phlegmatic). As in other cellular automata models, this is accomplished mathematically by biasing the probabilities that a bird will change from scanning to eating. In particular, let $T_{s}$ be the social temperature with values ranging from zero to infinity. The probability of a bird deciding to eat is then given by 


$$
P_{\text {scan }}=\frac{\left(n_{\text {eat }} / N\right)^{1 / T_{S}}}{\left(n_{\text {scan }} / N\right)^{1 / T_{s}}+\left(n_{\text {eat }} / N\right)^{1 / T_{S}}}
$$

where $N=8$ is the total number of neighbours that the bird is capable of processing, $n_{\text {scan }}$ is the number of neighbours that are scanning, and $n_{\text {eat }}=N-n_{\text {scan }}$ is the number of neighbours that are eating. (Note that on the periphery of the flock, $n_{\text {eat }}$ is still defined as $N-n_{\text {scan }}$ although it may not correspond with the number of neighbours eating because fewer than $N$ neighbours may be present; see the above discussion of the model assumptions.) The probability of deciding to eat is given by $P_{\text {eat }}=1-P_{\text {scan }}$. Note that when $T_{s}=1$, the probability of scanning reduces to $N_{\text {eat }} / N$, just as in the model described and used above. When the temperature approaches zero, the equation reduces to the majority rule model described above; without using probabilities, the birds choose their actions by doing the opposite of the majority. At zero temperature, a scanning bird cannot be persuaded to eat until a majority of its neighbours are also scanning. On the other hand, when $T_{s}$ is large (much greater than 1 ), the probabilities approach one-half, and the bird is likely to change from scanning to eating even if only one neighbour is scanning (i.e. it was easily persuaded by this one neighbour). A more complete description of temperature in cellular automatons can be found in Bahr \& Passerini (1998a, b).

When temperature is included in the bird flock simulations, higher temperatures (with more easily persuaded birds) create a less coordinated scan/feed pattern. At lower temperatures, when the birds are harder to persuade, the flock has a more pronounced and coordinated scan/feed pattern and the patches within the pattern last longer (growing and shrinking more slowly). At higher temperatures, the patches tend to shrink and disappear. Essentially, at higher temperatures, the birds become hypersensitive to their neighbours and will take any opportunity to change their action. Because the birds change actions so easily (almost randomly), the coordinated scan/feed pattern is disrupted (and less obvious) at higher temperatures.

Temperature has been introduced to the model because it has such a strong influence on coordinated scan/feed behaviour. Although coordinated scan/feed behaviour is apparent when studying video footage of evening grosbeaks (data from Bekoff 1995), the patterns are typically subtle or noisy. This suggests that the social temperature of the grosbeak flocks is high, so that some coordination is present but the scan/feed pattern is less obvious. Additional field observations could quantify the extent of the coordinated scan/feed pattern by measuring the average correlation between every two birds in a flock (two birds are correlated if they are both feeding or if they are both scanning). When the temperature is high, the patterns will be less obvious and the correlations will disappear. In fact, by standard techniques used in statistical physics, the correlation can be linked directly to the temperature (e.g. Ma 1985; Huang 1987). Thus, the degree of observed scan/feed coordination could be used to measure the temperature of bird flocks.

\section{Nondimensionality of Parameter Values}

The model does not specify the number of minutes or seconds associated with each time step or the fraction of a metre that separates each bird on the grid. Similarly, the social temperature and frequency with which birds look up from eating are model parameters that have not been assigned units or observationally derived values. Without these values the model is nondimensional and predicts flock behaviours in arbitrary units. For example, the simulated time spent scanning (Figs 2,3) will not correlate with the values of real numbers from empirical studies until realistic dimensions and values are assigned to each model parameter.

Rather than assigning units and reproducing the behaviour of one particular flock of one bird species, our simulations have focused instead on a more general test of the underlying behaviour. We have sought to 
demonstrate that the basic vigilance behaviour of birds is adequately described by a small set of local interactions. To do this we used a variety of parameter values and abstracted relevant relationships between parameters and vigilance behaviour (e.g. lower temperatures mean more coordinated behaviour). Given the variety of observations reproduced in this general sense, the model appears to be successful. Given this success, it would be reasonable to attempt field studies that pin down specific social temperatures (and other parameters) under specific situations that need to be modelled.

Figure 5. An illustration of the scan/feed pattern for a large flock $\left(T_{s}=0.9\right)$. A flock of 841 birds is unusual but is useful as a model result that easily illustrates the pattern without video images. (a) Initial random arrangement of scanning (white) and feeding (black) birds. Grey cells are boundaries at the edge of the flock. (b) Arrangement of the birds after 49 time steps. Note that the arrangement is no longer random, and birds are clumped into patches of feeding and scanning. (c) At the next time step (50), the birds have swapped eating and scanning roles. This swap continues from time step to time step, but the patches will evolve in shape and size. In fact, some small changes are visible between time steps 49 and 50 . This scan/feed pattern can be visually striking in videos that display many time steps in succession.
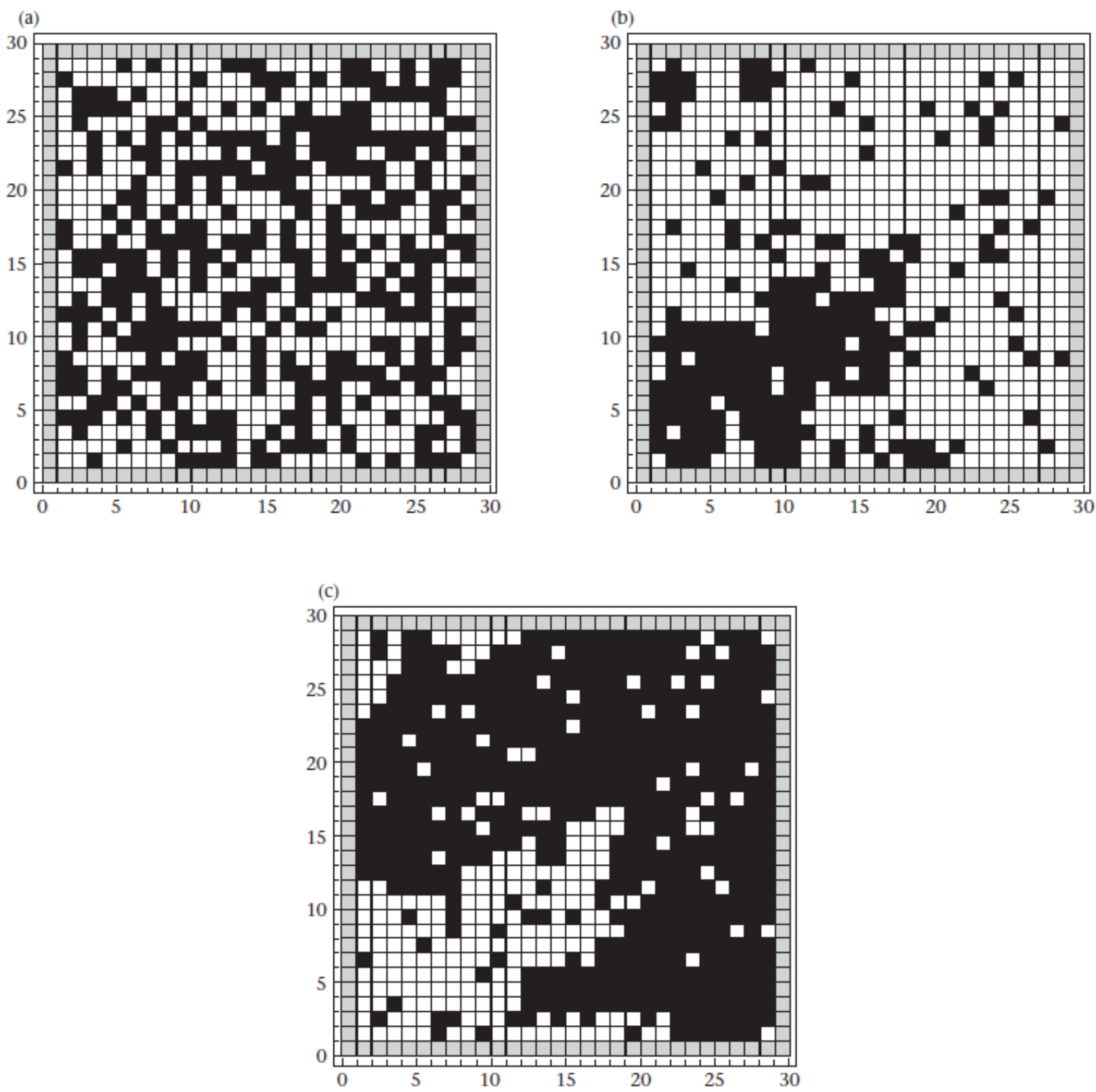


\section{DISCUSSION}

Vigilant behaviours in a flock can be reproduced in a numerical model by implementing a very simple set of interactions between neighbouring birds. A bird decides whether to scan or to eat based on the number of its neighbours that are doing each behaviour. If more neighbours are scanning, then a bird will decide to eat. If more neighbours are eating, then a bird will decide to scan. More complicated interactions and more complicated models might give additional details of flock behaviours, but might also obscure the fundamental reasons for the observed coordinated behaviours. Because of the model's simplicity, we know that birds in a flock do not have to see every other bird in a flock to determine when and how much time they should spend scanning. All that a bird needs to know is gathered from nearest neighbours, and information about other (hidden) birds in the flock is passed to them indirectly through a chain of neighbours. The simplicity of the model also increases our confidence in the results.

Although the model is not complicated, a number of well-known observations about vigilance are still predicted. For example, increasing flock size implies decreasing time spent scanning. The percentage of time spent scanning falls off asymptotically with flock size (as demonstrated by for example Bekoff 1995; Sadedin \& Elgar 1998). Flock geometry influences scan times, and birds in a line, for example, spend more time scanning than the same number of birds arranged in a circle or a square (e.g. Metcalfe 1984; Elgar 1989; Bekoff 1995).

\section{Practical Illustrations of the Model}

The numerical model's utility, however, goes well beyond confirming established observations. The model also predicts behaviours that can be confirmed with additional field studies. Our numerical experiments have not exhausted all possible predictions and the following three examples are only illustrative. (1) Scan time increases steadily as the flock geometry 'flattens out' from a square shape to a rectangle to a line, with flock size fixed (Bekoff 1995). In essence, the more birds that are on the perimeter of the flock, the more time the average bird in the flock will spend scanning. This is a direct consequence of the assumptions. (2) Birds trade turns scanning and eating in a coordinated spatial pattern (Bekoff 1995), and a measure of persuasion or social temperature influences this coordinated scan/feed behaviour. The more easily persuaded the birds (higher temperature), the less coordinated is their behaviour. (3) The more frequently the birds look up from eating to check on their neighbours, the more coordinated becomes the flock's scan/feed pattern. In fact, this interscan interval has been explored in many other models (for a review see Bednekoff \& Lima 1998), and those observations and results could enhance future cellular automata flock simulations.

To demonstrate its ability to predict empirically observed behaviours, our modeling efforts have focused on flocks of birds. However, schools of fish, colonies of ants, packs of wolves, herds of elk, antelope, buffalo, and many other types of animal groups also have local interactions between closest neighbours (Callen \& Shapero 1974; Bahr \& Passerini 1998a, b). The same type of cellular automaton model described here could explain the behaviour of these many diverse groups, and this cellular automaton approach could be used in a wide range of comparative studies that could lead to a greater understanding of many bird and other animal behaviours.

\section{Acknowledgments}

We thank two anonymous referees and Jeffrey Lucas for helpful comments on this paper. 


\section{References}

Alados, C. L. 1985. An analysis of vigilance in the Spanish ibex (Capra pyrenaica). Zeitschrift für Tierpsychologie, 68, 58-64.

Bahr, D. B. \& Passerini, E. 1998a. Statistical mechanics of opinion formation and collective behaviour: micro-sociology. Journal of Mathematical Sociology, 23, 1-27.

Bahr, D. B. \& Passerini, E. 1998b. Statistical mechanics of collective behaviour: macro-sociology. Journal of Mathematical Sociology, 23, 29-49.

Bednekoff, P. A. \& Lima, S. L. 1998. Randomness, chaos and confusion in the study of antipredator vigilance. Trends in Ecology and Evolution, 13, 284-287.

Bednekoff, P. A. \& Ritter, R. 1994. Vigilance in Nxai Pan springbok Antidorcas marsupialis. Behaviour, 128, 1-11.

Bekoff, M. 1995. Vigilance, flock size, and flock geometry: information gathering by western evening grosbeaks (Aves, Fringillidae). Ethology, 99, 150-161.

Bekoff, M. 1996. Cognitive ethology, vigilance, information gathering, and representation: who might know what and why? Behavioral Processes, 35, 225-237.

Bingman, V. P. 1993. Vision, cognition, and the avian hippocampus. In: Vision, Brain, and Behaviour in Birds (Ed. by H. P. Zeigler \& H. J. Bischof), pp. 391-408. Cambridge, Massachusetts: MIT Press.

Burger, J. \& Gochfeld, M. 1988. Effects of group size and sex on vigilance in ostriches (Struthio camelus): antipredator strategy or mate competition? Ostrich, 59, 14-20.

Burger, J. \& Gochfeld, M. 1994. Vigilance in African mammals: differences among mothers, other females and males. Behaviour, 131, 153-169.

Callen, E. \& Shapero, D. 1974. A theory of social imitation. Physics Today, July, 23-28.

Elgar, M. A. 1989. Predator vigilance and group size in mammals and birds: a critical review of the empirical evidence. Biological Reviews, 64, 13-33.

Elgar, M. A., Burren, P. J. \& Posen, M. 1984. Vigilance and perception of flock size in foraging house sparrows Passer domesticus L. Behaviour, 90, 215-223.

Emmerton, J. \& Delius, J. D. 1993. Beyond sensation. Visual cognition in pigeons. In: Vision, Brain, and Behaviour in Birds (Ed. by H. P. Zeigler \& H. J. Bischof), pp. 377-390. Cambridge, Massachusetts: MIT Press.

Hamilton, W. D. 1971. Geometry of the selfish herd. Journal of Theoretical Biology, 31, 295-311.

Huang K. 1987. Statistical Mechanics. 2nd edn. New York: J. Wiley. Hunter, L. T. B. \& Skinner, J. D. 1998. Vigilance behaviour in African ungulates: the role of predation pressure. Behaviour, 135, 195-211.

Krams, I. 1998. Dominance-specific vigilance in the great tit. Journal of Avian Biology, 29, 55-60.

Latané, B. 1981. The psychology of social impact. American Psychology, 36, 343-356.

Lima, S. L. 1990. The influence of models on the interpretation of vigilance. In: Interpretation and Explanation in the Study of Animal Behaviour, Vol. II: Explanation, Evolution, and Adaptation (Ed. by M. Bekoff \& D. Jamieson), pp. 246-267. Boulder, Colorado: Westview Press.

Ma, S. 1985. Statistical Mechanics. Philadelphia: World Scientific.

Metcalfe, N. B. 1984. The effect of habitat on the vigilance of shorebirds: is visibility important? Animal Behaviour, 32, 981-985.

Pepperberg, I. 1990. Some cognitive capacities of an African grey parrot (Psittacus erithacus). Advances in the Study of Behavior, 19, 357-409.

Pepperberg, I. 1994. Numerical competence in an African grey parrot (Psittacus erithacus). Journal of Comparative Psychology, 108, 36-44.

Quenette, P. Y. 1990. Functions of vigilance behaviour in mammals: a review. Acta Oecologica, 11, 801818.

Roberts, G. 1997. How many birds does it take to put a flock to flight? Animal Behaviour, 54, 1517-1522. 
Sadedin, S. R. \& Elgar, M. A. 1998. The influence of flock size and geometry on the scanning behaviour of spotted turtle doves, Streptopelia chinensis. Australian Journal of Ecology, 23, 177-180.

Speakman, A. 1987. Place cells in the brain: evidence for a cognitive map. Science Progress, Oxford, 71, 511-530.

Treves, A. 1998. The influence of group size and neighbours on vigilance in two species of arboreal monkeys. Behaviour, 135, 1-29.

Wolfram, S. 1986. Theory and Applications of Cellular Automata. Singapore: World Scientific.

Young, D. 1984. A local activator-inhibitor model of vertebrate skin patterns. Mathematical Biosciences, 72, 51-58. 